\title{
Clinical algorithm for malaria during low and high transmission seasons
}

\author{
Lulu Muhe, Birhane Oljira, Hirut Degefu, Fikre Enquesellassie, Martin W Weber
}

\begin{abstract}
Objectives-To assess the proportion of children with febrile disease who suffer from malaria and to identify clinical signs and symptoms that predict malaria during low and high transmission seasons. Study design-2490 children aged 2 to 59 months presenting to a health centre in rural Ethiopia with fever had their history documented and the following investigations: clinical examination, diagnosis, haemoglobin measurement, and a blood smear for malaria parasites. Clinical findings were related to the presence of malaria parasitaemia.
\end{abstract}

Results-Malaria contributed to $5.9 \%$ of all febrile cases from January to April and to $30.3 \%$ during the rest of the year. Prediction of malaria was improved by simple combinations of a few signs and symptoms. Fever with a history of previous malarial attack or absence of cough or a finding of pallor gave a sensitivity of $83 \%$ in the high risk season and $75 \%$ in the low risk season, with corresponding specificities of $51 \%$ and $60 \%$; fever with a previous malaria attack or pallor or splenomegaly had sensitivities of $80 \%$ and $69 \%$ and specificities of $65 \%$ and $81 \%$ in high and low risk settings, respectively.

Conclusion-Better clinical definitions are possible for low malaria settings when microscopic examination cannot be done. Health workers should be trained to detect pallor and splenomegaly because these two signs improve the specificity for malaria.

(Arch Dis Child 1999;81:216-220)

Keywords: developing countries; malaria; epidemiology; clinical signs

Malaria contributes to about 1 million childhood deaths worldwide each year, mostly in sub-Saharan Africa. ${ }^{1}$ In sub-Saharan Africa, children with fever are generally treated with antimalarial drugs without laboratory confirmation of parasitaemia. ${ }^{2}$ Even though most of sub-Saharan Africa belongs to the malaria hyperendemic or holoendemic areas, the actual risk of malaria transmission varies considerably: it is often much lower in urban areas, in semi-arid zones, in highland fringe areas, and during the dry season. The proportion of children correctly diagnosed with malaria varies according to the prevalence and whether it is the high transmission or low transmission season. ${ }^{3}$ Because malaria is a serious, potentially life threatening disease, it is customary in high transmission seasons or highly endemic areas to treat all children with febrile disease with an antimalarial drug when laboratory diagnosis is not available. ${ }^{4}$ During high transmission seasons, overtreatment of children with fever but without parasitaemia with antimalarial drugs could be avoided by the use of better clinical definitions. ${ }^{5}$ However, the problem is worse in settings with a low risk of transmission, where malaria accounts for only a small proportion of febrile cases, and a presumptive diagnosis of malaria results in enormous over treatment with an antimalarial, increased risk of rapid development of resistance, and increased risk of misdiagnosis of other febrile illnesses, such as typhoid fever and relapsing fever. ${ }^{6}$ In the current recommendation in the algorithm for the "integrated management of childhood illnesses" (IMCI) developed by WHO and UNICEF, ${ }^{7}$ the febrile child from a low risk malaria setting is treated for malaria if there is no running nose or measles. However, there are insufficient data to support the above recommendation.

Therefore we planned our study to assess the proportion of children presenting to a health care facility with febrile disease who have malaria, and to identify clinical signs and symptoms that predict malaria in contrast to other febrile diseases during low and high transmission seasons.

\section{Methods}

STUDY SETTING

The study site is located in the densely populated Butajira district, $130 \mathrm{~km}$ south of Addis Ababa, Ethiopia. The topography of Butajira comprises highland, lowland, and in between characteristics with altitude ranging between 1500 and $2300 \mathrm{~m}$ above sea level. The town of Butajira where the study health centre was situated lies at an altitude of $2000 \mathrm{~m}$ and, therefore, at the border of the malaria endemic area. There is usually very little transmission of malaria during the first four months of the year, January to April. Both Plasmodium falciparum and Plasmodium vivax are prevalent in the district.

\section{PATIENT ENROLMENT}

Children between 2 and 59 months of age, who presented at the outpatient department with fever (citing either a recent history of fever and/or a recorded rectal temperature of $38^{\circ} \mathrm{C}$ or above) in the morning working hours of the day were eligible for the study. All children with fever were registered on a logbook where a research nurse entered their weight, height, residence, and diagnosis. A systematic sample 
Table 1 Variables documented during history and physical examination

Background information
Age in months
Sex
Child coming from a known malarious village
Previous attack of malaria
Recent travel to known malarious village
History of current illness
Fever pattern (intermittent/continuous)
Diarrhoea (watery/bloody)
Chills/rigors
Earache
Running nose
Poor feeding/failure to suck
Vomiting
History of convulsion
Cough
Urinary problems (increased frequency/dysuria/change in
colour)
Difficulty in breathing
Physical examination
Respiratory rate
Wheeze
Total liver span
Pulse rate
Stridor
Tenderness of liver
Pallor (conjunctival/palmar/nail bed/tongue)
Bronchial breathing
Splenomegaly
Exudates on tonsils
Reduced air entry
Dehydration signs
Tympanic membrane (red/bulging/discharge)
Neck vein congestion
Altered consciousness
Grunting
Pulsations over the neck
Tone
Nasal flaring
Gallop rhythm
Peripheral oedema
Chest indrawing
Ejection systolic murmur
Skin rash (urticaria/scarlet fever/measles/scabies/pyoderma)
Crepitations
Hepatomegaly

of these children (every other child) was enrolled. Enrolled children had a detailed history and clinical examination performed by a study physician. A total of 59 variables was defined and documented (table 1). To maintain the quality of the clinical data, in addition to the intensive initial training and the pilot study, the senior investigators saw the same patients during their weekly visits and filled the same forms independently. The cases were discussed afterwards and discrepancies resolved.

\section{LABORATORY TESTS}

A finger prick blood sample for haematocrit, malarial parasites, and Borrelia recurrentis was obtained for thick and thin film examination. Blood films were stained with 4\% Giemsa stain and examined for 100 microscopic thick film fields under an oil immersion objective before being declared negative. Densities were recorded as the number of parasites $/ 200$ white blood cells. Using a standard mean white blood cell count of $8000 / \mu 1$, the densities were converted to the number of parasites $/ \mu 1$ of blood. ${ }^{8}$ Serum, cerebrospinal fluid, and/or urine samples were obtained from children with a clinical suspicion of sepsis, typhus, typhoid, meningitis, or a urinary tract infection. The physician recorded the immediately available laboratory results on the patient's form and made a diagnosis. Sample collection was carried out over one whole year to include both high and low risk malaria seasons. To maintain the quality of laboratory results, all blood films were taken in two containers and the tests were repeated centrally in Addis Ababa. Discrepancies of the blood film results were resolved by discussions by the laboratory technicians. Repeated presentations of the same patients with the same problem were excluded. The investigators ensured that appropriate measures were taken for sterilisation of equipment to avoid transmission of microorganisms by syringes and needles or pricking materials, in accordance with the recommendation adopted by the WHO Global Programme on AIDS.

\section{CLINICAL DEFINITIONS}

In the categorisation of febrile disease, the following case definition was used-malaria: any degree of parasitaemia of plasmodium was considered positive. The species of plasmodium ( $P$ vivax or $P$ falciparum) was determined. A second cut off point of 10000 parasites $/ \mu \mathrm{l}$ was used in a second analysis because it has been suggested that it reflects clinical malaria better. ${ }^{9}$

Treatment was according to standard practice. Very sick children were referred to the nearest hospital. Informed consent was obtained from the caretakers or guardians.

\section{ANALYSIS}

Data were entered into EpiInfo version 6 program. The frequency of malaria cases, as defined above, versus other febrile illnesses was compared for every month and season, and low versus high transmission seasons for malaria were defined. Frequencies were compared using the $\chi^{2}$ test. The odds ratios for signs and symptoms taken singly were evaluated, defining malaria by any parasitaemia and by parasitaemia $>10000 / \mu 1$ of blood. Logistic regression was used to assess the independent contribution of the signs and symptoms. Sensitivity and specificity of significant signs and symptoms or combinations were computed for both high risk and low risk seasons. Analysis was done using EpiInfo and Stata software packages.

\section{Results}

Our study was conducted over a period of 12 months during the calendar year 1996. Of 2540 children aged between 2 months and 5 years who were enrolled, 2490 had a complete examination including laboratory investigations. Of these, $730(29.3 \%)$ were less than 12 months of age, and $552(22.2 \%)$ were between 12 months and 2 years old. There were 1322 (53.1\%) boys and $1168(46.9 \%)$ girls. Eighty three children $(3.3 \%)$ required admission to the health centre and of these nine needed further referral to a hospital $90 \mathrm{~km}$ away. There was only one child who died during the follow up period after enrolment (diagnosis of malaria and severe pneumonia). Of the 2490 children, $120(5 \%)$ were re-examined by senior clinicians to check the reproducibility of clinical findings. 


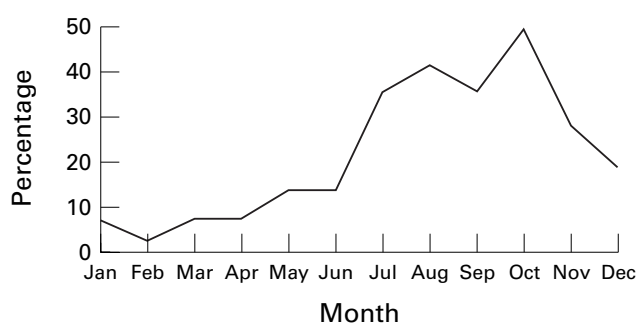

Figure 1 Seasonal pattern of malarial prevalence. The graph shows the percentage of children studied with a positive blood smear.

Table 2 Proportion of malaria and other common causes of fever in the cohort during the two seasons fanuary to April and May to December 1996

\begin{tabular}{|c|c|c|c|}
\hline & $\begin{array}{l}\text { Low risk season } \\
\text { fanuary-April }\end{array}$ & $\begin{array}{l}\text { High risk Season } \\
\text { March-December }\end{array}$ & \\
\hline Cause of fever & Number (\%) & Number (\%) & Total (\%) \\
\hline Malaria` & $48(6.0)$ & $511(30.3)$ & $559(22.4)$ \\
\hline $\begin{array}{l}\text { Other cause } \\
\text { of fever }\end{array}$ & $756(94.0)$ & $1175(69.7)$ & 1931 (77.6) \\
\hline Total & $804(32.3)$ & $1686(67.7)$ & $2490(100)$ \\
\hline
\end{tabular}

Every month, between 190 and 253 children were enrolled into the study. There were 804 children $(32.3 \%)$ enrolled during the low malaria transmission season (January to April, hereafter referred to as low risk), whereas there were 1686 children $(67.7 \%)$ enrolled during the high malaria transmission season (hereafter referred to as high risk) (fig 1). Of the children with febrile illnesses other than malaria, there were 36 cases of relapsing fever, 71 cases of measles, and 10 cases of typhoid fever; for all three infections there was no significant differ- ence in incidence between low and high risk malaria seasons. There were 695 children with pneumonia contributing to $27.9 \%$ of the enrolled children $(38 \%$ during low risk and $22 \%$ during high risk malaria seasons). There were 665 patients with diarrhoea, accounting for $26.7 \%$ of all enrolled children (45\% during low risk and $16 \%$ during high risk malaria seasons). Figure 1 shows the proportion of malaria cases among the children for every month of the year. There were 48 cases of malaria with a mean monthly prevalence of $5.9 \%$ in the low risk season, and a total of 511 malaria cases with a mean monthly prevalence of $30.3 \%$ in the high risk season. During this season the prevalence of malaria increased to reach a peak of $50 \%$ in October and then declined. The difference in the prevalence of malaria between the two periods of the year was significant (table 2). There were 233 children $(41.7 \%)$ with $P$ vivax malaria, 326 children $(58.3 \%)$ with $P$ falciparum malaria, and four were infected with both species of plasmodium. During the low transmission season, $47.2 \%$ had $P$ vivax and during the high transmission season $41.2 \%$ had $P$ vivax (not significant).

Using univariate analysis, the odds ratio to malaria for all symptoms and signs was computed and those with a significant association are shown in table 3 . When malaria was defined as parasitaemia $>10000$, only three cases were found during the low risk season and 69 cases during the high risk season. Therefore, analysis using this definition was only done for the high risk season. When malaria was defined as any parasitaemia, 28 signs and symptoms were identified to be

Table 3 Number of children with clinical findings and univariate analysis results for clinical findings associated with any degree of parasitaemia of malaria in the low risk season and with any degree of parasitaemia in addition to parasitaemia $\geqslant 10$ 000/ul in the high risk season among 2490 children studied

\begin{tabular}{|c|c|c|c|c|c|c|}
\hline & \multicolumn{4}{|c|}{ High risk season } & \multirow{2}{*}{\multicolumn{2}{|c|}{$\begin{array}{l}\text { Low risk season } \\
\text { Parasitaemia }>0(n=48)\end{array}$}} \\
\hline & \multicolumn{2}{|c|}{ Parasitaemia $>0(n=511)$} & \multicolumn{2}{|c|}{ Parasitaemia $>10000(n=69)$} & & \\
\hline & $n$ & Odds ratio $(95 \% \mathrm{CI})$ & $n$ & Odds ratio $(95 \% \mathrm{CI})$ & $n$ & Odds ratio $(95 \%$ CI) \\
\hline Malarious area & 431 & $1.94(1.57$ to 2.40$)$ & 58 & $1.98(0.99 \text { to } 4.06)^{\star \star}$ & 35 & $3.96(2.13$ to 7.37$)$ \\
\hline Travel history & 213 & $2.29(2.00$ to 2.63$)$ & 43 & $5.74(3.37$ to 9.81$)$ & 1 & $0.33(0.05 \text { to } 2.37)^{\star \star}$ \\
\hline Previous attack & 160 & 1.89 (1.63 to 2.18$)$ & 17 & $1.39(0.76-2.52)^{\star \star}$ & 21 & $4.74(2.77$ to 8.08$)$ \\
\hline Intermittent fever & 390 & $1.70(1.39$ to 2.08$)$ & 52 & $1.43(1.12$ to 2.37$)$ & 48 & $2.16(0.69 \text { to } 6.83)^{\star \star}$ \\
\hline Chills/rigors & 226 & $2.70(2.36$ to 3.09$)$ & 38 & 4.48 (2.67 to 7.55$)$ & 11 & $2.92(1.56$ to 5.49$)$ \\
\hline Running nose & 78 & $0.67(0.54$ to 0.83$)$ & 7 & $0.41(0.17$ to 0.95$)$ & 14 & $0.36(0.20$ to 0.66$)$ \\
\hline Diarrhoea & 153 & $0.72(0.61$ to 0.85$)$ & 21 & $0.71(0.41 \text { to } 1.24)^{\star \star}$ & 17 & $0.50(0.28$ to 0.88$)$ \\
\hline Earache & 42 & $0.71(0.54$ to 0.93$)$ & 7 & $0.89(0.36 \text { to } 2.07)^{\star \star}$ & 3 & $0.20(0.06$ to 0.62$)$ \\
\hline Poor feeding & 366 & $0.83(0.71$ to 0.98$)$ & 36 & $0.46(0.27$ to 0.78$)$ & 36 & $0.96(0.51 \text { to } 1.80)^{\star \star}$ \\
\hline Convulsion & 51 & $2.24(1.87$ to 2.69$)$ & 7 & $2.42(0.97 \text { to } 5.76)^{\star \star}$ & 0 & $\star$ \\
\hline Cough & 248 & $0.60(0.52$ to 0.69$)$ & 36 & $0.59(0.35$ to 0.99$)$ & 20 & $0.55(0.31$ to 0.95$)$ \\
\hline Difficult breathing & 253 & $1.51(1.31$ to 1.75$)$ & 45 & $3.01(1.76$ to 5.17$)$ & 13 & $0.51(0.27$ to 0.94$)$ \\
\hline Pallor & 275 & $2.83(2.46$ to 3.25$)$ & 33 & $2.32(1.38$ to 3.88$)$ & 17 & $5.39(3.14$ to 9.27$)$ \\
\hline Tonsillar exudates & 23 & $0.34(0.23$ to 0.50$)$ & 1 & $0.10(0.00$ to 0.65$)$ & 1 & $0.39(0.06 \text { to } 2.79)^{\star \star}$ \\
\hline Red/bulging ear drum & 27 & $0.68(0.49$ to 0.97$)$ & 1 & $0.17(0.01 \text { to } 1.18)^{\star \star}$ & 1 & $0.51(0.07 \text { to } 3.59)^{\star \star}$ \\
\hline Fast breathing & 261 & $1.24(1.07$ to 1.44$)$ & 43 & $1.58(1.34$ to 1.84$)$ & 21 & $0.84(0.45 \text { to } 1.5)^{\star \star}$ \\
\hline Grunting & 153 & $1.23(1.05$ to 1.44$)$ & 22 & $1.36(0.78 \text { to } 2.37)^{\star \star}$ & 3 & $0.57(0.18 \text { to } 1.79)^{\star \star}$ \\
\hline Chest indrawing & 25 & $0.53(0.37$ to 0.76$)$ & 2 & $0.29(0.05 \text { to } 1.25)^{\star \star}$ & 5 & $0.49(0.20 \text { to } 1.22)^{\star \star}$ \\
\hline Crepitations & 44 & $0.37(0.28$ to 0.49$)$ & 4 & $0.23(0.07$ to 0.67$)$ & 8 & $0.49(0.23 \text { to } 1.03)^{\star \star}$ \\
\hline Pulsations over neck & 6 & $2.23(1.40$ to 3.56$)$ & 0 & $\star$ & 1 & $0.98(0.14 \text { to } 6.71)^{\star \star}$ \\
\hline Gallop rythmn & 9 & $1.77(1.13$ to 2.79$)$ & 1 & $1.49(0.00 \text { to } 11.04)^{\star \star}$ & 0 & $\star$ \\
\hline Systolic murmur & 8 & $2.24(1.49$ to 3.36$)$ & 1 & $2.17(0.00 \text { to } 16.81)^{\star \star}$ & 1 & $5.67(1.12$ to 28.75$)$ \\
\hline Hepatomegaly & 201 & $2.69(2.36$ to 3.07$)$ & 36 & $4.92(2.92$ to 8.28$)$ & 1 & $1.87(0.29 \text { to } 12.15)^{\star \star}$ \\
\hline Liver tenderness & 115 & $2.52(2.20$ to 2.89$)$ & 25 & $5.59(3.20$ to 9.71$)$ & 1 & $4.33(0.78 \text { to } 24.20)^{\star \star}$ \\
\hline Splenomegaly & 256 & 3.65 (3.20 to 4.14$)$ & 42 & $6.16(3.62$ to 10.45$)$ & 12 & 14.91 (9.42 to 23.62$)$ \\
\hline Dehydration signs & 32 & $0.47(0.29$ to 0.76$)$ & 32 & $0.74(0.46$ to 0.98$)$ & 3 & $0.46(0.11 \text { to } 1.86)^{\star \star}$ \\
\hline Peripheral oedema & 26 & $1.63(1.23$ to 2.18$)$ & 2 & $0.89(0.00 \text { to } 3.88)^{\star \star}$ & 1 & $0.98(0.14 \text { to } 6.71)^{\star \star}$ \\
\hline Measles rash & 3 & $0.21(0.07$ to 0.63$)$ & 1 & $0.76(0.00 \text { to } 5.39)^{\star \star}$ & 1 & $0.23(0.03 \text { to } 1.80)^{\star \star}$ \\
\hline
\end{tabular}

* Odds ratios not computed because there were no children with the clinical finding.

$\star \star$ Non-significant odds ratios, all others are significant.

CI, confidence interval. 
Table 4 Logistic regression analysis for clinical findings independently associated to any degree of parasitaemia in addition to parasitaemia $\geqslant 10$ 000/ $\mathrm{l}$ in high risk season and any degree of parasitaemia of malaria in low risk season among 2490 children studied

\begin{tabular}{llll}
\hline & \multicolumn{1}{l}{ High risk season } & & Low risk season \\
\cline { 2 - 3 } $\begin{array}{lll}\text { Parasitaemia }>0 \\
\text { Pdds ratio }(95 \% \text { CI) }\end{array}$ & $\begin{array}{l}\text { Parasitaemia } \geqslant 10000 \\
\text { Odds ratio }(95 \% \text { CI) }\end{array}$ & $\begin{array}{l}\text { Parasitaemia }>0 \\
\text { Odds ratio }(95 \% \text { CI) }\end{array}$ \\
\hline Travel history & $1.76(1.22$ to 2.52$)$ & $1.17(0.06$ to 3.63$)$ & $0.05(0.01$ to 1.00$)$ \\
Previous attack & $1.46(1.06$ to 2.01$)$ & $2.28(1.34$ to 3.89$)$ & $2.80(1.19$ to 6.54$)$ \\
Chills/rigors & $2.75(2.04$ to 3.70$)$ & $0.62(0.27 \text { to } 1.45)^{\star}$ & $1.61(0.62 \text { to } 4.18)^{\star}$ \\
Running nose & $0.67(0.46$ to 0.98$)$ & $1.08(0.60$ to 1.95$)$ & $0.46(0.19 \text { to } 1.08)^{\star}$ \\
Diarrhoea & $0.85(0.64 \text { to } 1.14)^{\star}$ & $2.62(1.03$ to 6.66$)$ & $0.34(0.15$ to 0.75$)$ \\
Earache & $1.07(0.67 \text { to } 1.73)^{\star}$ & $0.65(0.38 \text { to } 1.13)^{\star}$ & $0.05(0.01$ to 0.42$)$ \\
Cough & $0.56(0.41$ to 0.75$)$ & $1.79(1.00$ to 3.25$)$ & $1.07(0.48 \text { to } 2.41)^{\star}$ \\
Difficult breathing & $1.66(1.17$ to 2.36$)$ & $1.08(0.62 \text { to } 1.89)^{\star}$ & $1.07(0.43 \text { to } 2.67)^{\star}$ \\
Pallor & $2.02(1.39$ to 2.94$)$ & $0.26(0.03$ to 1.95$)$ & $4.69(1.52$ to 14.5$)$ \\
Tonsillar exudates & $0.44(0.26$ to 0.72$)$ & $1.92(1.06$ to 3.63$)$ & $0.27(0.02 \text { to } 3.89)^{\star}$ \\
Splenomegaly & $3.12(2.20$ to 4.44$)$ & & $42.11(5.58$ to 317.7$)$ \\
\hline
\end{tabular}

^Non-significant odds ratios, all others are significant.

$\mathrm{CI}$, confidence interval.

significantly associated with malaria in the high risk season. When malaria was defined by the presence of parasitaemia $\geqslant 10000 / \mu 1$, only 15 of these signs and symptoms were significantly associated. Eleven of the 28 parameters were found to be significantly associated with any parasitaemia in the low risk season. During the high risk season, some of these, such as the presence of runny nose, diarrhoea, earache, poor feeding, cough, exudative tonsils, red or bulging ear drums, chest wall indrawing, crepitations, signs of dehydration, or a measles rash, were found to be negatively associated, indicating that malaria was less likely in their presence. Other clinical signs, such as coming from a known malarious area, previous attack of malaria, intermittent fever, chills or rigors, convulsions, difficulty in breathing, pallor, fast breathing, grunting, pulsations over the neck, a gallop rhythm, a heart murmur, hepatomegaly, a tender liver, splenomegaly, and peripheral oedema, were significantly positively associated risk factors. The clinical findings significantly negatively associated with parasitaemia during the low risk season were a runny nose, diarrhoea, earache, cough, and difficulty in breathing, whereas positively associated findings were patient coming from a malarious area, a previous malaria attack, chills or rigors, pallor, a systolic heart murmur, and splenomegaly (table 3 ).

When the significantly associated clinical findings found in the univariate analysis were

Table 5 Sensitivity and specificity of individual or combinations of clinical findings in children with fever independently associated with any degree of parasitaemia of malaria in low or high risk seasons among 2490 children studied

\begin{tabular}{llllll}
\hline & \multicolumn{2}{l}{ High risk } & & & \multirow{2}{*}{ Low risk } \\
\cline { 2 - 3 } \cline { 5 - 6 } & Sensitivity & Specificity & & Sensitivity & Specificity \\
\hline Travel history & 41.7 & 83.8 & & 2.1 & 93.8 \\
Previous attack & 31.3 & 85.8 & & 43.8 & 87.3 \\
Chills/rigors & 44.2 & 86.4 & & 22.9 & 91.7 \\
No running nose & 84.7 & 23.9 & & 70.8 & 54.5 \\
No diarrhoea & 70.1 & 40.9 & & 64.6 & 55.8 \\
No earache & 91.8 & 12.6 & & 93.8 & 27.0 \\
No cough & 48.5 & 69.4 & & 41.7 & 72.5 \\
No difficult breathing & 50.5 & 35.3 & & 27.1 & 56.6 \\
Pallor & 53.8 & 81.6 & & 35.4 & 92.5 \\
No tonsillar exudates & 95.5 & 16.2 & & 97.9 & 5.3 \\
Splenomegaly & 50.1 & 90.4 & & 25.5 & 99.2 \\
No running nose or no measles (IMCI) & 84.2 & 26.1 & 70.8 & 56.4 \\
Previous attack or pallor or no cough & 82.8 & 51.4 & 75.0 & 59.5 \\
Previous attack or pallor or splenomegaly & 80.0 & 68.5 & & 64.6 & 81.2
\end{tabular}

IMCI, WHO/UNICEF programme of integrated management of childhood illnesses. used in a logistic regression analysis, only a few remained significant (table 4). During the low risk season, six findings were significantly associated with malaria (positively or negatively). During the high risk season, nine findings were found to be significantly associated with malaria defined as any parasitaemia. When these signs were analysed for heavy parasitaemia ( $\geqslant 10000 / \mu \mathrm{l})$ running nose, cough, and previous attack of malaria became nonsignificant and earache became significant.

The sensitivity and specificity of those signs and symptoms that were found to be significantly associated with malaria using the logistic regression analysis either for the high risk or the low risk season were computed as shown in table 5. None of these signs individually had both sensitivity and specificity $>60 \%$. The current IMCI recommendation as applied for the low risk setting had a sensitivity of $71 \%$ and a specificity of $56 \%$. A combination of fever, previous attack of malaria, or pallor or no cough gave a $75 \%$ sensitivity and $60 \%$ specificity for the low risk setting. A combination of fever, previous attack of malaria, or pallor or splenomegaly raised the specificity to $81 \%$ and the sensitivity was nearly $65 \%$ for the low risk setting. The latter combination gave the best sensitivity and specificity $(80 \%$ and $69 \%$, respectively) for the high risk setting.

\section{Discussion}

Because microscopy for the diagnosis of malaria is often unavailable in peripheral health facilities in developing countries, our study aimed to identify a few clinical features that are both sensitive and specific for the diagnosis of malaria by low level health workers. Thus, even though we started out with 59 clinical findings, some of which were probably inappropriate for training of low level health workers, we have found a few simple clinical findings and combinations that can be used to improve the prediction of malaria in children with fever and to guide initiation of treatment. These features are particularly important during low transmission seasons, but it was interesting to note that they performed similarly in both high and low season. Because during high malaria transmission some children might be expected to have a low asymptomatic parasitaemia, a threshold of 
$10000 / \mu$ of blood (which has been suggested to reflect clinical malaria better) was also used for analysis. ${ }^{4}$

As suggested by the WHO, our study used the presence of a history of fever or documented fever by the health worker as an entry point for considering malaria. Our studies confirm that measured temperature at the time of presentation to a health facility is not a useful criterion by itself, because only $25 \%$ of our cases had a recorded fever. Surveys of health facilities in Cote d'Ivoire ${ }^{10}$ and Angola ${ }^{11}$ showed that body temperature was measured in only $68 \%$ and $2 \%$, respectively, of children who were brought to clinic because of fever. Therefore, we feel that a recent history of fever is an adequate reason to suspect malaria and to investigate the child further.

Addition of pallor and splenomegaly increased the specificity of this all encompassing definition considerably. However, standardisation and training of health workers to assess children for pallor can be difficult. Previous studies that have used pallor to diagnose anaemia have had mixed results. ${ }^{12-16}$ Health workers in high risk malaria areas are likely to see pallor so often that they learn to detect it more reliably. ${ }^{17}$ More effort to train health workers in low risk areas to detect pallor might be worthwhile. Grover has suggested that health workers need additional training to detect splenomegaly, ${ }^{18}$ although interobserver variability and insensitivity have been reported. ${ }^{19}$ However, a recent study from the Gambia suggests that splenomegaly can be detected reproducibly by staff with limited training. ${ }^{17}$ Our finding of an association between fever and splenomegaly and malaria is supported by other studies. ${ }^{50-23}$

In low risk settings, detecting another cause of fever reduces the likelihood of malaria. Thus, the absence of other obvious causes of infection such as running nose or cough or diarrhoea gave a reasonably high sensitivity and specificity. Because the number of measles cases was small in our study, the inclusion of absence of measles was not very useful. Although signs of pneumonia were also negatively associated with malaria, it should be noted that a considerable overlap between the presentation of pneumonia and malaria has been reported elsewhere. ${ }^{24}$

Our analysis has not tried to look at the clinical predictors for the different species of malaria or at the impact of the presence of other diagnoses such as pneumonia, typhoid fever, or relapsing fever. These diagnoses require better training of staff and/or additional laboratory tests, which are not available in many peripheral health facilities.

In summary, we found that the specificity of the diagnosis of malaria can be increased without great sacrifice to sensitivity by using a combination of a few simple clinical findings. The most useful clinical signs are pallor and an enlarged spleen. Better clinical definitions like these can result in economy in antimalarial drug use, which may reduce the speed of emergence of resistance to such drugs. However, there will always be a compromise between sensitivity and specificity. Particularly in low transmission seasons or settings, investment in facilities for microscopy might be worthwhile. ${ }^{25}$ Our results might be useful in settings where microscopic examination cannot be performed, as is currently the case in most of subSaharan Africa.

This project was funded by Special Programme for Research and Training in Tropical Diseases (TDR) grant no 940890 . We are grateful to the physicians, nurses, laboratory technicians, and other staff of the Butajira Health Centre for their cooperation; in particular, Drs M Ahmed, M Wondewossen, K Bereda, tion; in particular, Drs M Ahmed, $M$ Wondewossen, K Bereda,
A Kebede, G Baruda, and W Eshetu. We also thank Drs J CatA Kebede, G Baruda, and W Eshetu. We also thank Drs J Cat-
tani and S Gove from TDR/WHO and CHD/WHO, respectani and S Gove from TDR/WHO and CHD/WHO, respec-
tively, for their advice and support in the initiation of the study. tively, for their advice and support in the initiation of the study.
We thank Mr K Woldeyesus for entering and cleaning the data. We thank Mr K Woldeyesus for entering and cleaning the data.
We are indebted to the parents of the many children who conWe are indebted to the parents of
sented to be enrolled in the study.

1 World Bank. World development report 1993. Investing in World Bank. World development report 1993.

2 Rougemont A, Breslow N, Brenner E, et al. Epidemiological basis for clinical diagnosis of childhood malaria in endemic zone in West Africa. Lancet 1991;338:1292-5.

3 Olivar M, Develoux M, Chegou Abari A, Loutan L. Presumptive diagnosis of malaria results in a significant risk of mis-treatment of children in urban Sahel. Trans $R$ Soc Trop Med Hyg 1991;85:729-30.

4 Genton B, Smith T, Baea K, et al. Malaria: how useful are clinical criteria for improving the diagnosis in a highly endemic area. Trans $R$ Soc Trop Med Hyg 1994;88:537-41.

5 Redd SC, Kazembe PN, Luby SP, et al. Clinical algorithm for treatment of plasmodium

6 Daniel E, Beyene H, Tessema T. Relapsing fever in children-demographic, social and clinical features. Ethiop Med f 1992;30:207-14.

7 WHO. Integrated management of childhood illness. Geneva: World Health Organisation, Division of Child Health and Development, 1995.

8 Greenwood BM, Armstrong JRM. Comparison of two simle methods for determining malaria parasite density. Trans R Soc Trop Med Hyg 1991;85:186-8.

9 Armstrong Schellenberg JRM, Smith T, Alonso PL, Hayes RJ. What is clinical malaria? Finding case definitions for field research in highly endemic areas. Parasitol Today 1994;10:439-42.

10 Traore, M. Evaluation de l'impact des formations et des besoins en formation des personnels de sante. Rapport final. Abidjan, Cote d'Ivoire: Ministry of Health, 1991

11 Bjork M, Johansson R, Kanji N. Improving the quality of primary care services in Angola. Health Policy and Planning 7. 1992:290-5.

12 Gjorup T, Bugge PM, Hendriksen C, Jensen AM. A critical evaluation of the clinical diagnosis of anemia. Am f Epidemiol 1986;124:657-65.

13 Nardone DA, Roth KM, Mazur DJ, McAfee JH. Usefulness of physical examination in detecting the presence or absence of anemia. Arch Intern Med 1990;150:201-4.

14 Strobach RS, Anderson SK, Doll DC, Ringenberg QS. The value of the physical examination in the diagnosis of anemia. Correlation of the physical findings and the hemoglobin concentration. Arch Intern Med 1988;148:831-2.

15 Wurapa FK, Bulsara MK, Boatin BA. Evaluation of conjunctival pallor in the diagnosis of anaemia. F Trop Med Hyg 1986;89:33-6.

16 Sanchez-Carrillo CI. Bias due to conjunctiva hue and the clinical assessment of anemia. F Clin Epidemiol 1989;42: 751-4.

17 Weber MW, Kellingray SD, Palmer A, Jaffar S, Mulholland EK, Greenwood BM. Pallor as a clinical sign of severe anaemia in children: an investigation in the Gambia. Bull World Health Organ 1997;75(suppl 1):113-18.

18 Grover SA, Barkun AN, Sackett DL. Does this patient have splenomegaly? ҰAMA 1993;270:2218-21.

19 Tamayo SG, Rickman LS, Mathews WC, et al. Examiner dependence on physical diagnostic tests for the detection of dependence on physical diagnostic tests for the detection of
splenomegaly: a prospective study with multiple observers. splenomegaly: a prospective study

20 Greenwood BM. Asymptomatic malaria infections-do they matter? Parasitol Today 1987;3:206-14.

21 Delfini LF. The relationship between body temperature and malaria parasitaemia in rural forest areas of Western Nigeria. F Trop Med Hyg 1973;76:111-14

22 Olaleye BO, Williams LA, D'Alessandro U, et al. Clinical predictors of malaria in Gambian children with fever or a history of fever. Trans $R$ Soc Trop Med Hyg 1998;98:300-4.

23 Luxemburger C, Nosten F, Kyle DE, Kiricharoen L, Chongsuphajaisiddhi T, White NJ. Clinical features cannot predict a diagnosis of malaria or differentiate the infecting ppecies in children living in an area of low transmission. Trans R Soc Trop Med Hyg 1998;92:45-9.

24 O'Dempsey TJ, McArdle TF, Laurence BE, et al. Overlap in the clinical features of pneumonia and malaria in African children. Trans $R$ Soc Trop Med Hyg 1993;87:662-5.

25 Jonkman A, Chibwe RA, Khoromana CO, et al. Cost-saving through microscopy-based versus presumptive diagnosis of through microscopy-based versus presumptive diagnosis of
malaria in adult outpatients in Malawi. Bull World Health Organ 1995;73:223-7. 